\title{
Determinación de la frecuencia de hiperamilasemia y pancreatitis en pacientes llevados a colangiopancreatografía retrógrada endoscópica
}

\section{Determining Frequency of Hyperamylasemia and Pancreatitis in Patients after Endoscopic Retrograde Cholangiopancreatography}

\author{
Fabián Rodrigo Del Castillo Rangel' ${ }^{1}$ Lázaro Antonio Arango Molano²
}

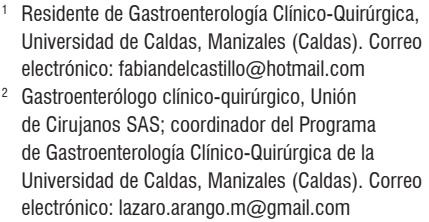

Residente de Gastroenterología Clínico-Quirúrgica, Universidad de Caldas, Manizales (Caldas). Correo electrónico: fabiandelcastillo@hotmail.com 2 Gastroenterólogo clínico-quirúrgico, Unión de Cirujanos SAS; coordinador del Programa de Gastroenterología Clínico-Quirúrgica de la Universidad de Caldas, Manizales (Caldas). Correo electrónico: lazaro.arango.m@gmail.com

Fecha recibido: $26-01-17$ Fecha aceptado: 28-07-17

\begin{abstract}
Resumen
Hemos visto con preocupación que, en el postquirúrgico de la colangiopancreatografía retrógrada endoscópica (CPRE), existe confusión en cuanto a la aparición de pancreatitis y a la elevación transitoria de las amilasas (hiperamilasemia sin repercusión clínica), por lo que nos dimos a la tarea de realizar esta investigación, cuyo objetivo principal era determinar la prevalencia del incremento de niveles séricos de amilasas y de pancreatitis en los pacientes sometidos a colangiopancreatografía retrógrada endoscópica, según variables demográficas, clínicas y procedimentales. Es un estudio descriptivo, prospectivo, analítico y observacional, la población de estudio estuvo integrada por 98 pacientes atendidos en Unión de Cirujanos SAS, quienes requirieron colangiopancreatografía retrógrada endoscópica.

Resultados: la frecuencia de pancreatitis aguda después de la CPRE fue del 2\%, en 2 de los 98 casos estudiados; el $30 \%$ presentó hiperamilasemia (30 pacientes); la canulación del conducto pancreático mostró asociación con pancreatitis post-CPRE, con un valor de $p<0,05$. De los 2 pacientes que presentaron pancreatitis post-CPRE, a uno de ellos se le realizó contraste del conducto pancreático. La dilatación con balón se asoció con la hiperamilasemia $(p<0,041)$.

Conclusiones: la pancreatitis post-CPRE se presentó en un $2 \%$ de los pacientes, y este porcentaje se encuentra incluido dentro de los pacientes que presentaron hiperamilasemia, uno de los criterios para el diagnóstico de pancreatitis. Nuestro grupo se encuentra en el rango inferior del promedio internacional $(1,8 \%$ al $7,2 \%$ ). La hiperamilasemia asintomática se presentó en un $30 \%$. Recomendamos que, si después de la CPRE, el paciente no presenta dolor, no se midan las amilasas, porque estas van a estar elevadas en gran número de los casos y, entonces, solo causaría confusión.
\end{abstract}

\section{Palabras clave}

Colangiopancreatografía retrógrada endoscópica, CPRE, coledocolitiasis, pancreatitis, hiperamilasemia, conducto pancreático.

\section{INTRODUCCIÓN}

La colangiopancreatografía retrógrada endoscópica (CPRE) es un procedimiento relativamente nuevo en nuestro medio, mediante el cual se pueden realizar el diagnóstico y el tratamiento de diversas patologías de origen pancreatobiliar, de carácter benigno o maligno. Entre las aplicaciones más frecuentes podemos encontrar: cálculos de la vía biliar (coledocolitiasis) o del conducto pancreático; estenosis biliares de origen benigno o maligno; colocación de prótesis para alivio temporal o paliativo de la ictericia y sus posibles complicaciones; drenaje de colangitis; estudio y toma de muestras directamente de la vía biliar con cepillos; y manejo paliativo de tumores periampulares (tumores de la cabeza del páncreas, el duodeno, la vía biliar distal y la ampolla de Vater). La CPRE tiene, 


\begin{abstract}
We have seen with concern that there is confusion regarding the appearance of pancreatitis and the transient elevation of amylases (hyperamylasemia without clinical repercussions) in the postoperative period following ERCP (endoscopic retrograde cholangiopancreatography). For this reason, we embarked on the task of determining the prevalence of increased serum amylases and pancreatitis in patients who have undergone endoscopic retrograde cholangiopancreatography according to demographic, clinical and procedural variables. This is a descriptive, prospective, analytical and observational study. The study population consisted of 98 patients treated in the Union of SAS Surgeons who required endoscopic retrograde cholangiopancreatography.

Results: Acute pancreatitis was found in $2 \%$ of the patients who had undergone ERCP (Two of the 98 cases studied). Thirty patients (30\%) presented hyperamylasemia. Cannulation of the pancreatic was associated with post-ERCP pancreatitis $(p<0.05)$. Pancreatic duct contrast had been used in one of the two patients who presented post-ERCP pancreatitis. Balloon dilation was associated with hyperamylasemia $(p<0.041)$.

Conclusions: Post-ERCP pancreatitis was found in two patients $(2 \%)$, both of whom also presented hyperamylasemia which is one of the criteria for diagnosis of pancreatitis. The rate in our group is at the lower end of the international range of averages from $1.8 \%$ to $7.2 \%$. Asymptomatic hyperamylasemia was present in $30 \%$ of our group. Following ERCP, we recommend that there is no need to measure amylases in patients who do not present pain. Amylase levels will be elevated in a large number of cases and will only cause confusion.

\section{Keywords}

Endoscopic retrograde cholangiopancreatography, ERCP, choledocholithiasis, pancreatitis, hyperamylasemia, pancreatic duct.
\end{abstract}

además, la ventaja de ser menos invasiva para el paciente, por cuanto no hay necesidad de realizar incisiones sobre la pared abdominal, lo que permite una recuperación más rápida del paciente y un retorno más temprano a su cotidianeidad. Además, se puede realizar bajo sedación en un tiempo corto, bien sea en quirófano o en una sala de endoscopia. El riesgo de pancreatitis post-CPRE "es más alto entre mujeres, pero estos hallazgos son confusos por la presencia de disfunción del esfínter de Oddi, la cual ocurre con mayor frecuencia en mujeres" (1).

Con respecto a esta técnica (CPRE), se ha incrementado su uso en instituciones de mediana y alta complejidad, razón por la cual también se han empezado a hacer más evidentes las complicaciones como pancreatitis, sangrado digestivo, perforaciones digestivas, entre las más frecuentes; y otras como embolia aérea, lesión biliar, fiebre y sepsis, especialmente cuando este procedimiento se utiliza con fines terapéuticos. Además, se presenta la dificultad de entender la contribución relativa y el efecto interactivo de los múltiples factores de riesgo, combinación que va aumentando el riesgo progresivamente. Según un estudio, "el riesgo de pancreatitis post-CPRE (PEP) se incrementó del 5\% en mujeres con niveles de bilirrubina normales hasta el $16 \%$ sumándole la canulación difícil y la sospecha de disfunción del esfínter de Oddi (DEO), que eleva el riesgo hasta el 42\%" (2).

La pancreatitis y la hiperamilasemia asintomática están muy asociadas con el procedimiento (CPRE); la primera es una entidad muy seria, mientras que la segunda tiene un mínimo peso estadístico, con una incidencia reportada de alrededor del 50\%. "La hidratación agresiva disminuye el síndrome de respuesta inflamatoria sistémica (SIRS), la severidad y las complicaciones en estudios experimentales y clínicos. Medir amilasas o lipasas de 4 a 6 horas después de la CPRE podría predecir el desarrollo de la PEP y ayudaría a decidir cuál paciente podría ser hospitalizado o cuál dado de alta el mismo día” (3).

La complicación más común de la CPRE es la pancreatitis, en muchas ocasiones sobrediagnosticada, con una incidencia que va desde el 1,8\% hasta el 7,2\% en la mayoría de las series prospectivas. Los criterios definidos para su diagnóstico están aceptados desde 1991 e incluyen el dolor abdominal de tipo pancreático, asociado con un incremento de por lo menos 3 veces el valor de referencia de amilasa o lipasa, y estos dos factores deben ocurrir en las 24 horas posteriores a la CPRE. La pancreatitis post-CPRE es una de las complicaciones más comunes y temidas dentro de los eventos adversos relacionados con la CPRE " $y$ resulta en una considerable morbilidad y, raramente, en la muerte. La tasa reportada de pancreatitis post-CPRE varía del $1 \%$ al $40 \%$, con un promedio del $5 \%$ al $7 \%$ que se ha observado en estudios retrospectivos y observacionales" $(4,5)$.

El dolor y los síntomas necesitan ser lo suficientemente severos para que requieran admisión al hospital o prolongación de la estancia hospitalaria actual. Aunque el $80 \%$ de los episodios de pancreatitis post-CPRE son leves, algunos pacientes desarrollarán pancreatitis severa y requerirán hospitalización prolongada en la unidad de cuidados inten- 
sivos y la utilización de una mayor cantidad de recursos hospitalarios. Los médicos y los pacientes deben tener en cuenta que no todo dolor seguido de CPRE constituye pancreatitis post-CPRE; también la hiperamilasemia transitoria sin pancreatitis aguda es común después de la CPRE. "La pancreatitis post-CPRE se define como un nuevo dolor abdominal o incremento de este que, clínicamente, corresponde al de la pancreatitis aguda (dolor típico en el epigastrio, irradiado a la espalda), asociado con elevación de amilasa en suero más de 3 veces de lo normal durante más de 24 horas después de la CPRE y que requiere ingreso hospitalario o prolongación de la hospitalización" (6).

Los factores de riesgo asociados con el paciente se han vinculado con alta tasas de pancreatitis post-CPRE en análisis multivariados. "Se incluye la edad joven (menor de 60 años), el sexo femenino, historia de pancreatitis post-CPRE previa, la falta de dilatación de los conductos, el nivel normal de bilirrubinas y la sospecha de disfunción del esfínter de Oddi. Asimismo, la pancreatitis crónica, particularmente la pancreatitis crónica calcificada y la malignidad pancreática, han mostrado tener un efecto protector para pancreatitis post-CPRE, al parecer por la disminución de la actividad enzimática exocrina y la atrofia del parénquima pancreático. La presencia de pancreas divisium, divertículo periampular, gastrectomía Billroth II, alergia al medio de contraste e intervenciones biliares en pacientes con esfinterotomía previa no están asociados con elevación del riesgo de pancreatitis post-CPRE” $(1,7)$.

A pesar de que la colangiopancreatografía retrógrada endoscópica es un procedimiento muy efectivo, también es invasivo por sus potenciales complicaciones, como la pancreatitis aguda, complicación que, en ocasiones, es muy severa. También se puede presentar la elevación de las enzimas amilasas de manera frecuente sin que exista una pancreatitis aguda como consecuencia de este procedimiento, pero esta última no es una complicación, sino un fenómeno causal por el procedimiento en sí. Al existir estos dos fenómenos, el de la pancreatitis aguda, que tendría que reunir varios elementos diagnósticos —entre ellos, hiperamilasemia más de 3 veces por encima del rango normal más dolor típico de pancreatitis y, además, que este persista durante un período mayor de 24 horas-, la hiperamilasemia sola ocurrirá con la elevación de las enzimas en sangre, ello corroborado por resultados de laboratorio, sin ningún curso clínico adicional en un postquirúrgico habitual. El entrenamiento del endoscopista depende del volumen y la experiencia. "Se ha sugerido que, de forma independiente, contribuye al riesgo de PEP, pero ha sido difícil de evaluar, $y$ el entrenamiento se ha mostrado como un factor que incrementa el riesgo de PEP (OR 1,5, IC del 95\%: 1,029$2,057 ; p=0,03)$. Presumiblemente, esto es resultado de una canulación traumática, la prolongación de una canulación dificultosa o el exceso en la utilización del electrocauterio durante la esfinterotomía" (8).

La dilatación con balón en un esfínter biliar intacto "se ha asociado con un riesgo marcadamente aumentado de pancreatitis post-CPRE” $(9,10)$. El uso de stents pancreáticos "ha mostrado la reducción de la incidencia y la severidad de PEP; el mecanismo es facilitar el drenaje del conducto pancreático aliviando la hipertensión que se desarrolla como resultado de la estenosis transitoria inducida durante el procedimiento" (11).

\section{MATERIALES Y MÉTODOS}

Este es un estudio descriptivo, prospectivo, analítico y observacional (Tabla 1). La población estuvo compuesta por los pacientes atendidos en Unión de Cirujanos SAS con patologías pancreatobiliares que requirieron CPRE. La población diana utilizada se constituyó con los pacientes sometidos a CPRE por el Grupo de Gastroenterología Clínico-Quirúrgica en el período de julio a septiembre del año 2016. La selección y el tamaño de la muestra respondió a la evaluación de la historia clínica, y los criterios de inclusión se limitaron a pacientes a quienes se les realizó CPRE por cualquier indicación, bajo seguimiento, que cumplieran con el consentimiento informado para las intervenciones requeridas. Se excluyeron los pacientes que presentaron complicaciones, como perforación, o que hubieran implicado cambios radicales, como manejos quirúrgicos abiertos.

Tabla 1. Distribución según el diagnóstico.

\begin{tabular}{|c|c|c|c|}
\hline Diagnóstico & Frecuencia & $\%$ & Acumulado \\
\hline Coledocolitiasis con colecistitis & 3 & $3,1 \%$ & $3,1 \%$ \\
\hline Coledocolitiasis sin colangitis & 60 & $61,2 \%$ & $64,3 \%$ \\
\hline Coledocolitiasis con colangitis & 1 & $1,0 \%$ & $65,3 \%$ \\
\hline Cáncer de vesícula & 1 & $1,0 \%$ & $66,3 \%$ \\
\hline Colangitis & 4 & $4,1 \%$ & $70,4 \%$ \\
\hline Colelitiasis & 3 & $3,1 \%$ & $73,5 \%$ \\
\hline Estenosis benigna vía biliar & 13 & $13,3 \%$ & $86,7 \%$ \\
\hline Estenosis maligna vía biliar & 9 & $9,2 \%$ & $95,9 \%$ \\
\hline Fístula biliar & 4 & $4,1 \%$ & $100,0 \%$ \\
\hline Total & 98 & $100,0 \%$ & \\
\hline
\end{tabular}

\section{RESULTADOS}

En total, el estudio incluyó a 98 pacientes que tenían indicación para realizar CPRE. La incidencia de pancreatitis post-CPRE fue del $2 \%$, con 1 paciente con pancreatitis 
leve y otro con pancreatitis severa, lo que da como resultado 2 pacientes en total con pancreatitis post-CPRE en todo el estudio. De los 96 pacientes restantes, ninguno presentó dicha afección (98\%). La incidencia de la hiperamilasemia fue del $30 \%$, en 30 pacientes; 28 de ellos con amilasas elevadas no tuvieron pancreatitis; tan solo 2 cursaron con ella. Cabe aclarar que estos son los mismos 2 pacientes en total que cursaron con pancreatitis. El sexo femenino representó el mayor porcentaje de la población (52 pacientes), que correspondió al 53\%. La procedencia del municipio de Manizales fue del 76\%. El diagnóstico lo representó la coledocolitiasis sin colangitis en 59 pacientes, el 60,2\% (Tablas 2, 3 y 4 ).

Tabla 2. Distribución según la pancreatitis.

\begin{tabular}{|c|c|c|c|c|}
\hline Pancreatitis & & Frecuencia & $\%$ & Acumulado \\
\hline Sin pancreatitis & & 96 & $98,0 \%$ & $98,0 \%$ \\
\hline Leve & & 1 & $1,0 \%$ & $99,0 \%$ \\
\hline \multirow[t]{2}{*}{ Severa } & & 1 & $1,0 \%$ & $100,0 \%$ \\
\hline & Total & 98 & $100,0 \%$ & \\
\hline
\end{tabular}

Tabla 3. Distribución según el sexo.

\begin{tabular}{|c|c|c|c|}
\hline Sexo & Frecuencia & $\%$ & Acumulado \\
\hline Masculino & 46 & $47,0 \%$ & $47,0 \%$ \\
\hline Femenino & 52 & $53,0 \%$ & $100,0 \%$ \\
\hline Total & 98 & $100,0 \%$ & \\
\hline
\end{tabular}

Tabla 4. Distribución según la hiperamilasemia.

\begin{tabular}{rcc}
\hline Hiperamilasemia & Frecuencia & Porcentaje \\
\hline Con hiperamilasemia & 30 & $30,6 \%$ \\
Sin hiperamilasemia & 68 & $69,3 \%$ \\
Total & $\mathbf{9 8}$ & $100 \%$ \\
\hline
\end{tabular}

Se realizó canulación del conducto pancreático a solo 9 pacientes (9\%); se realizó asociación estadística en los casos de pancreatitis con los pacientes a los que se les hizo canulación del conducto pancreático, con un valor de $\mathrm{p}<0,05$ (Tabla 5). A 2 pacientes que tuvieron pancreatitis, se les hizo canulación del conducto, y se realizó contraste del conducto pancreático a 6 pacientes $(6 \%)$. No se realizó a los 92 restantes (94\%): solo en uno de los dos casos de pancreatitis se efectuó el contraste del conducto pancreático. Es decir, en el 50\% de los casos, estimamos que no hubo una asociación estadística válida por el reducido número de casos, pero, si le damos importancia a que coincida con la mitad de los casos de enfermos en los que hubo dilatación con balón, hay una asociación estadística posi- tiva para los pacientes que presentaron hiperamilasemia $(\mathrm{p}<0,041)$ (Tabla 6).

Se realizó dilatación de la papila con balón a 7 pacientes, y no se hizo a los 91 restantes (Tabla 7). Entre quienes presentaron pancreatitis aguda, a ninguno se le realizó la dilatación con balón, pero debemos destacar que no es un procedimiento de rutina, como tampoco favorito en nuestro grupo debido al riesgo de producir potencialmente pancreatitis con el uso de esta técnica. El reducido número de casos analizados impide mostrar una relación estadística. Con respecto al precorte, se realizó a 21 pacientes (21\%), y a 77 no se les realizó (53\%); la papilotomía se realizó a 53 pacientes (54\%), pero no a 45 (46\%). No se demostró ninguna asociación estadística para pancreatitis o hiperamilasemia.

Las causas por las cuales los pacientes fueron sometidos a CPRE fueron las siguientes: coledocolitiasis sin colangitis, 60 pacientes $(61,2 \%)$; estenosis benigna de la vía biliar, 13 pacientes $(13,2 \%)$; estenosis maligna de la vía biliar, 9 pacientes $(9,2 \%)$; para fístula biliar, 4 pacientes cada uno $(4,1 \%)$; para coledocolitiasis con colecistitis y colelitiasis, 3 cada uno $(3,1 \%)$ y, para coledocolitiasis, coledocolitiasis con colangitis y cáncer de vesícula, uno para cada diagnóstico $(1,0 \%)$.

\section{DISCUSIÓN}

Nuestro trabajo muestra que la pancreatitis post-CPRE es una complicación que, si bien es frecuente, en nuestro grupo objeto fue del $2 \%$, lo que hace pensar que, en manos experimentadas y con alto nivel de entrenamiento, se puede encontrar en los promedios inferiores. La hiperamilasemia asintomática que no tiene implicaciones clínicas para el paciente es bastante frecuente (30\%). Recomendamos que, si después de la CPRE, el paciente no presenta dolor, no se midan las amilasas porque van a estar elevadas en gran número de los casos, lo que solo causaría confusión.

La canulación del conducto pancreático en relación con la aparición de la pancreatitis post-CPRE tuvo una asociación estadística significativa, con una $\mathrm{p}<0,05$, y apareció en todos los casos en los que se presentó la enfermedad.

No hubo asociación estadística en cuanto al contraste del conducto pancreático, que se presentó en 1 de los 2 casos que cursaron con pancreatitis aguda, el 50\% de los casos con pancreatitis. Sin embargo, sí otorgamos importancia a que este haya correspondido a la mitad de los casos. La dilatación con balón de la papila también demostró una asociación estadística significativa en la elevación de las amilasas, aunque no tuvo relación estadística con la pancreatitis, lo que podría deberse a que no es de uso rutinario en nuestro 
Tabla 5. Distribución según la presencia de pancreatitis y la canulación del conducto pancreático.

\begin{tabular}{|c|c|c|c|c|c|}
\hline \multicolumn{6}{|l|}{ Tabla cruzada } \\
\hline & & & \multicolumn{2}{|c|}{ Canulación del conducto pancreático } & \multirow[t]{2}{*}{ Total } \\
\hline & & & Sí & No & \\
\hline \multirow[t]{6}{*}{ Presencia de pancreatitis } & \multirow[t]{3}{*}{ Sí } & Recuento & 2 & 0 & 2 \\
\hline & & $\%$ dentro de presencia de pancreatitis & $100,0 \%$ & $0,0 \%$ & $100,0 \%$ \\
\hline & & $\begin{array}{l}\text { \% dentro de canulación del conducto } \\
\text { pancreático }\end{array}$ & $22,2 \%$ & $0,0 \%$ & $2,0 \%$ \\
\hline & \multirow[t]{3}{*}{ No } & Recuento & 7 & 89 & 96 \\
\hline & & $\%$ dentro de presencia de pancreatitis & $7,3 \%$ & $92,7 \%$ & $100,0 \%$ \\
\hline & & $\begin{array}{l}\% \text { dentro de canulación del conducto } \\
\text { pancreático }\end{array}$ & $77,8 \%$ & $100,0 \%$ & $98,0 \%$ \\
\hline \multirow[t]{3}{*}{ Total } & & Recuento & 9 & 89 & 98 \\
\hline & & $\%$ dentro de presencia de pancreatitis & $9,2 \%$ & $90,8 \%$ & $100,0 \%$ \\
\hline & & $\begin{array}{l}\% \text { dentro de canulación del conducto } \\
\text { pancreático }\end{array}$ & $100,0 \%$ & $100,0 \%$ & $100,0 \%$ \\
\hline \multicolumn{6}{|l|}{ Pruebas de chi cuadrado } \\
\hline & Valor & GI & $\begin{array}{c}\text { Significación } \\
\text { asintótica ( } 2 \text { caras) }\end{array}$ & $\begin{array}{l}\text { Significación } \\
\text { exacta ( } 2 \text { caras) }\end{array}$ & $\begin{array}{l}\text { Significación } \\
\text { exacta ( } 1 \text { cara) }\end{array}$ \\
\hline Chi cuadrado de Pearson & $20,190^{a}$ & 1 & 0,000 & & \\
\hline Corrección de continuidad ${ }^{b}$ & 10,604 & 1 & 0,001 & & \\
\hline Razón de verosimilitud & 9,991 & 1 & 0,002 & & \\
\hline Prueba exacta de Fisher & & & & 0,008 & 0,008 \\
\hline Asociación lineal por lineal & 19,984 & 1 & 0,000 & & \\
\hline N..$^{\circ}$ de casos válidos & 98 & & & & \\
\hline
\end{tabular}

a. Dos casillas (50,0\%) han esperado un recuento menor de 5. El recuento mínimo esperado es 18.

b. Solo se ha calculado para una tabla $2 \times 2$.

grupo por el alto riesgo de pancreatitis post-CPRE $y$, también, debido al reducido número de casos de la muestra. En cuanto al precorte, se les realizó a 21 pacientes (21\%) y a 75 (78\%) no se les realizó; a ninguno de los pacientes que tuvieron pancreatitis se les realizó precorte. En cuanto a la realización de papilotomía de rutina, no se presentó ninguna asociación estadísticamente significativa entre la hiperamilasemia o la pancreatitis aguda.

Hay evidencia que sugiere cómo el acceso al precorte de manera temprana, después de 5 a 10 intentos de canulación, puede disminuir el riego de PEP, comparado con los persistentes intentos para canular, lo que traumatiza la papila. Dos metaanálisis que incluyeron 6 estudios randomizados controlados, que compararon el precorte temprano con múltiples intentos por canular mediante un abordaje estándar, mostraron una significativa reducción en la PEP (OR
0,47, IC del 95\%: 0,24-0,91) cuando se realizó el precorte temprano; además, se pudo evidenciar que el precorte también redujo el tiempo de realización de la CPRE, lo cual podría ser beneficioso en muchos pacientes que son de edad avanzada y que tienen una escala de ASA elevada.

\section{CONCLUSIONES}

Consideramos que la canulación, el contraste del conducto pancreático y la dilatación de la papila son factores de riesgo que podrían estar relacionados con la presencia de pancreatitis aguda y que pueden modificarse. La realización de un precorte temprano ante una canulación difícil podría ser un factor protector, lo que puede ser modificable siempre y cuando la CPRE la realice un profesional con entrenamiento. 
Tabla 6. Distribución según la presencia de pancreatitis y el contraste del conducto pancreático.

\begin{tabular}{|c|c|c|c|c|c|}
\hline \multicolumn{6}{|l|}{ Tabla cruzada } \\
\hline & & & \multicolumn{2}{|c|}{ Contraste del conducto pancreático } & \multirow[t]{2}{*}{ Total } \\
\hline & & & Sí & No & \\
\hline \multirow{6}{*}{ Presencia de pancreatitis } & \multirow{3}{*}{ Sí } & Recuento & 1 & 1 & 2 \\
\hline & & $\%$ dentro de presencia pancreatitis & $50,0 \%$ & $50,0 \%$ & $100,0 \%$ \\
\hline & & $\begin{array}{l}\% \text { dentro de contraste conducto } \\
\text { pancreático }\end{array}$ & $16,7 \%$ & $1,1 \%$ & $2,0 \%$ \\
\hline & \multirow{6}{*}{ No } & Recuento & 5 & 91 & 96 \\
\hline & & $\%$ dentro de presencia pancreatitis & $5,2 \%$ & $94,8 \%$ & $100,0 \%$ \\
\hline & & $\begin{array}{l}\% \text { dentro de contraste conducto } \\
\text { pancreático }\end{array}$ & $83,3 \%$ & $98,9 \%$ & $98,0 \%$ \\
\hline \multirow{3}{*}{ Total } & & Recuento & 6 & 92 & 98 \\
\hline & & $\%$ dentro de presencia pancreatitis & $6,1 \%$ & $93,9 \%$ & $100,0 \%$ \\
\hline & & $\begin{array}{l}\% \text { dentro de contraste conducto } \\
\text { pancreático }\end{array}$ & $100,0 \%$ & $100,0 \%$ & $100,0 \%$ \\
\hline \multicolumn{6}{|c|}{ Pruebas de chi cuadrado } \\
\hline & Valor & GI & $\begin{array}{c}\text { Significación } \\
\text { asintótica ( } 2 \text { caras) }\end{array}$ & $\begin{array}{l}\text { Significación } \\
\text { exacta ( } 2 \text { caras) }\end{array}$ & $\begin{array}{l}\text { Significación } \\
\text { exacta ( } 1 \text { cara) }\end{array}$ \\
\hline Chi cuadrado de Pearson & $6,839^{a}$ & 1 & 0,009 & & \\
\hline Corrección de continuidad ${ }^{b}$ & 1,266 & 1 & 0,251 & & \\
\hline Razón de verosimilitud & 3,087 & 1 & 0,079 & & \\
\hline Prueba exacta de Fisher & & & & 0,119 & 0,119 \\
\hline Asociación lineal por lineal & 6,769 & 1 & 0,009 & & \\
\hline N. ${ }^{\circ}$ de casos válidos & 98 & & & & \\
\hline
\end{tabular}

a. Dos casillas (50,0\%) han esperado un recuento menor de 5. El recuento mínimo esperado es 12.

b. Solo se ha calculado para una tabla $2 \times 2$.

Tabla 7. Distribución según el nivel de amilasa y los rangos de dilatación de la papila.

\begin{tabular}{lcccc}
\hline Rangos & & & \\
\hline & Dilatación de la papila & N. ${ }^{0}$ & Rango promedio & Suma de rangos \\
\hline \multicolumn{1}{c}{ Nivel de amilasa } & Sí & 7 & 70,64 & 494,50 \\
& No & 91 & 47,87 & 4356,50 \\
& & 98 & \\
\hline Estadísticos de prueba ${ }^{a}$ & Nivel de amilasa & & \\
\hline & 170,500 & & \\
U de Mann-Whitney & 4356,500 & & \\
W de Wilcoxon & $-2,043$ & & \\
Z & 0,041 & & \\
Sig. asintótica (bilateral) & & \\
\hline
\end{tabular}

\section{REFERENCIAS}

1. Masci E, Mariani A, Curioni S, et al. Risk factors for pancreatitis following endoscopic retrograde cholangiopancreatography: a meta-analysis. Endoscopy. 2003;35:830-4. Doi: https://doi.org/10.1055/s-2003-42614
2. Freeman ML, DiSario JA, Nelson DB, et al. Risk factors for post-ERCP pancreatitis: a prospective, multicenter study. Gastrointest Endosc. 2001;54:425-34. Doi: https://doi. org/10.1067/mge.2001.117550

3. Tener S, Baillie J, Dewitt J, et al. Management of acute pancreatitis. American College of Gastroenterology guideline. Am J Gastroenterol. 2013;108:1400-15. 
4. Bakman; Freeman ML. Update on biliary and pancreatic sphincterotomy. Curr Opin Gastroenterol. 2012;28:420-6.

5. Andriulli A, Loperfido S, Napolitano G, et al. Incidence rates post ERCP complications: a systematic survey of prospective studies. Am J Gastroenterol. 2007;102:1781-8. Doi: https://doi.org/10.1111/j.1572-0241.2007.01279.x

6. Cotton PB, Lehman G, Vennes JA. Endoscopic sphincterotomy complications and their management: an attempt at consensus. Gastrointest Endosc. 1991;37:383-91. Doi: https://doi.org/10.1016/S0016-5107(91)70740-2

7. Freeman ML. Complications of endoscopic retrograde cholangiopancreatography: avoidance and management. Gastrointest Endosc Clin N Am. 2012;22:567-86. Doi: https://doi.org/10.1016/j.giec.2012.05.001

8. Cheng CL, Sherman S, Watkins JL, et al. Risk factors for post ERCP pancreatitis: a prospective multicenter study.
Am J Gastroenterol. 2006;101:139-47. Doi: https://doi. org/10.1111/j.1572-0241.2006.00380.x

9. Disario JA, Freeman ML, Bjorkman DJ, et al. Endoscopic balloon dilation compared with sphincterotomy for extraction of bile duct stones. Gastroenterology. 2004;127:12919. Doi: https://doi.org/10.1053/j.gastro.2004.07.017

10. Weinberg BM, Shindy W, Lo S. Endoscopic balloon sphincter dilation (sphincteroplasty) versus sphincterotomy for common bile duct stones. Cochrane Database Syst Rev. 2006;4:cd004890.

11. Elmunzer BJ, Waljee AK, Elta GH, et al. A meta-analysis of rectal NSAIDs in the prevention of post-ERCP Pancreatitis. Gut. 2008;57:1262-7. Doi: https://doi.org/10.1136/ gut.2007.140756 